EGFR-Blockade

\title{
Neue Therapieoption für solide Tumoren
}

\begin{abstract}
Auf außergewöhnlich großes Interesse stieß auf dem diesjährigen Deutschen Krebskongress in Berlin ein Satellitensymposium zum Thema Anti-EGFR-Therapie. In der von Merck ausgerichteten Veranstaltung stellten Experten die Einsatzmöglichkeiten des neuen monoklonalen Antikörpers Cetuximab vor. Die Ergebnisse der ersten Studien wurden von den Meinungsbildnern als äußerst viel versprechend eingestuft.
\end{abstract}

Der Epidermal Growth Factor Receptor (EGFR) spielt nach aktueller Auffassung für das Wachstum solider Tumoren eine herausragende Rolle. Es handelt sich um einen transmembranären Rezeptor, der zur Gruppe der Human Epidermal Growth Factor Receptors (HER) gehört. Seine intrazelluläre Domäne besitzt eine Tyrosinkinaseaktivität.

Seine wichtigsten Liganden sind der Epidermal Growth Factor (EGF) sowie der Transforming Growth Factor alpha (TGF- $\alpha$ ).

Bindet ein Ligand an den EGFR, so bildet dieser mit einem zweiten aktivierten
EGFR oder einem andern Rezeptor der HER-Familie ein Homo- oder Heterodimer. Die folgende Tyrosinkinase-Aktivierung setzt verschiedene Signaltransduktionswege in Gang, die für das Überleben der Zelle wichtig sind. Dadurch werden Zellteilung, Zelldifferenzierung, Zellüberleben, Angiogenese sowie die Zelladhäsion reguliert (Abb. 1).

\section{Pathologische Überaktivierung}

Hinsichtlich des Tumorwachstums richtet der EGFR immer dann Schaden an, wenn es zu einer übermäßigen Aktivierung kommt. Dazu ist eine Überexpression nicht zwingend nötig. Auch bei normaler Rezeptordichte kann es zu einer Überaktivierung kommen, dann nämlich, wenn einer der Liganden im Übermaß vorhanden ist. Ursache hierfür kann die Produktion von EGF oder TGF- $\alpha$ durch den Tumor selbst sein. Man spricht dann von einem autokrinen Loop. Außerdem ist auch eine konstitutive Aktivierung, bei der der Rezeptor mutiert und dadurch
Abb. 1. Über die Signalkaskade des EGF-Rezeptors werden neben der Zellproliferation auch weitere Schlüsselprozesse der Tumorgenese aktiviert.

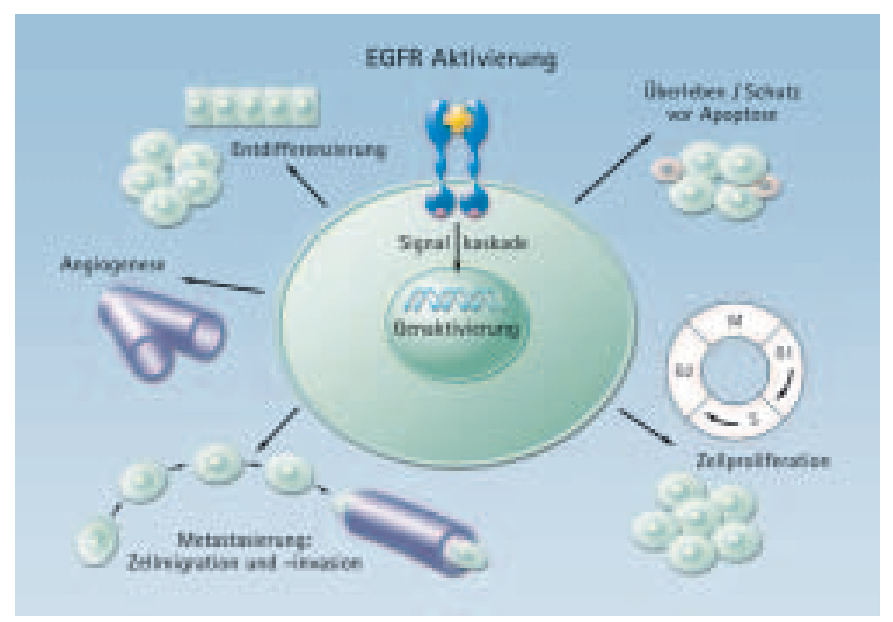

auch ohne das Vorhandensein eines Liganden aktiviert wird, möglich.

Wie Professor Dr. Wolfgang E. Berdel von der Medizinischen Klinik und Poliklinik A der Universität Münster berichtete, wurde die Bedeutung des EGFR für das Tumorwachstum schon 1984 erkannt. Viele solide Tumore exprimieren den EGFR. Zu ihnen gehören das Kolorektalkarzinom, das Bronchialkarzinom, Kopf und Halstumore sowie Magen- und Ovarialkarzinome. Für die meisten dieser Tumoren ist die EGFR Expression mit einer ungünstigen Prognose assoziiert.

Professor Berdel leitet aus diesen Erkenntnissen ab, dass es sich beim EGFR um ein wichtiges Ziel bei der Tumorbekämpfung handelt. Bereits seit 1983 steht ein gegen den EGFR gerichteter monoklonaler Maus-Antikörper (M225) zur Verfügung. Dieser erwies sich in präklinischen Experimenten als effektiv, versagte allerdings in klinischen Studien, da ein großer Prozentsatz der Probanden humane Anti-MausAntikörper (HAMA) bildete, die diesen Antikörper sofort neutralisierten.

\section{Zwei Drittel Mensch, ein Drittel Maus}

Es wurde daher ein chimärer Antikörper entwickelt, bei dem nur noch die variablen Anteile von der Maus stammen, während die konstanten Regionen human sind. Die neue Entwicklung bekam den Namen Cetuximab. Cetuximab erzeugt nur bei zirka $4 \%$ der $\mathrm{Pa}$ tienten HAMA-Reaktionen, die aber klinisch nicht relevant sind, und bindet mit hoher Affinität an die extrazelluläre Komponente des EGFR. Eine Bindung des Liganden wird dadurch zuverlässig verhindert, und die Aktivierung der Tyrosinkinase unterbleibt. Zusätzlich scheint die Bindung von Cetuximab an den Rezeptor eine Rezeptorinternalisierung und eine antikörperbedingte Zytotoxizität zu induzieren. In präklinischen Studien zeigte sich eine zytostatische Wirksamkeit bei Kolonkarzinomen, Kopf/Hals-Tumoren, Blasenkrebs, Brustkrebs und Nierenkarzinomen. Außerdem wurde eine additive Wirkung bei gleichzeitiger Gabe von Chemotherapeutika oder Bestrahlung beobachtet.

\section{Die BOND-Studie beim Kolorektal- karzinom}

Die beste Datenlage zu Cetuximab liegt derzeit für das metastasierende Kolorek- 
talkarzinom vor. Nachdem kleinere Pilotstudien viel versprechend verlaufen waren, wurde die randomisierte BONDStudie (Bowel Oncology with Cetuximab Antibody) durchgeführt. An ihr nahmen 300 Patienten mit EGFR-positivem Kolorektalkarzinom im Stadium IV teil. Alle Studienteilnehmer wiesen innerhalb von maximal 3 Monaten nach Irinotecan-basierter Chemotherapie einen progredienten Krankheitsverlauf auf. Während der BOND-Studie erhielten sie entweder Cetuximab alleine $(\mathrm{n}=100)$ oder in Kombination mit Irinotecan $(n=200)$.

Die alleinige Gabe von Cetuximab führte bei $32,4 \%$ der Patienten zu einem Ansprechen, während die kombinierte Gabe eine Ansprechrate von 55,5\% zeigte. Als Ansprechen wurde dabei sowohl eine Teilremission (PR) als auch eine Krankheitsstabilisierung (SD) gewertet. Die mittlere Zeit bis zur Krankheitsprogression (TTP) betrug in der Cetuximab-Gruppe 1,5 und in der Kombinationsgruppe 4,1 Monate. Das mediane Überleben (OS) war mit 6,9 Monaten unter Monotherapie und 8,6 Monaten unter Kombination mit Irinotecan nicht signifikant unterschiedlich.

«Cetuximab ist die erste Substanz, bei der wir zeigen können, dass sie eine Resistenz einer Chemotherapie überwinden kann», kommentierte Prof. Claus-Henning Köhne von der Medizinischen Klinik und Poliklinik I des Universitätsklinikums Dresden die von ihm vorgestellten Ergebnisse. Interessant war für ihn außer- dem die Tatsache, dass das Ansprechen auf Cetuximab weder mit der Höhe der EGFR-Expression noch mit der Anzahl oder Art der vorausgegangenen Chemotherapien korrelierte. Aus den Ergebnissen der Studie leitet er die in Abbildung 2 dargestellten Einsatzmöglichkeiten für Cetuximab ab.

\section{Verträglichkeit von Cetuximab}

Verglichen mit herkömmlichen Chemotherapien ist Cetuximab extrem gut verträglich. Auffälligste Nebenwirkung ist ein akneähnlicher Hautausschlag. Sein Auftreten und seine Schwere sind mit dem Ansprechen auf Cetuximab korreliert. Die Nebenwirkung wird von den Patienten im Allgemeinen gut toleriert, insbesondere dann, wenn sie darüber informiert sind, dass der Ausschlag ein gutes Zeichen für die Wirksamkeit ist und dass er im Verlauf der Therapie nachlässt und meist völlig verschwindet.

Mittlerweile liegen auch erste Pilotstudien zum Einsatz von Cetuximab in First-lineTherapie vor. Die Ergebnisse erachtet Prof. Köhne für so gut, dass er größere Studien für wünschenswert hält.

\section{Weitere Ziele des monoklonalen Antikörpers}

Erste Studien gibt es mittlerweile auch zum Einsatz von Cetuximab bei KopfHals-Karzinomen und beim nicht-kleinzel-

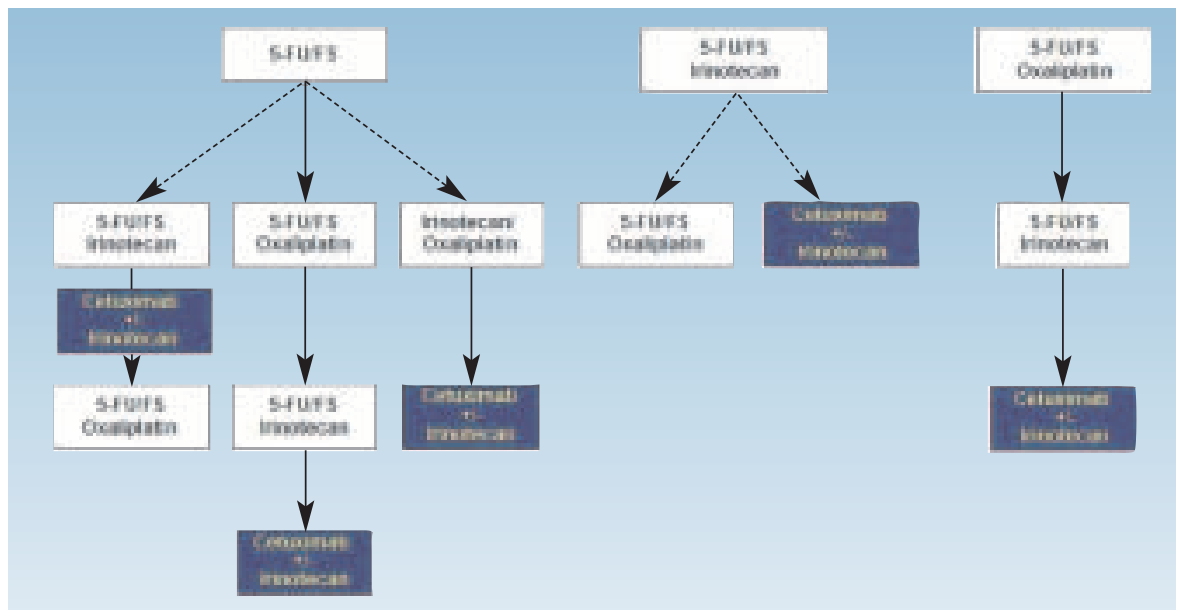

Abb. 2. Aufgrund der guten Erfahrungen mit Cetuximab in der BOND-Studie sieht Prof. Köhne beim metastasierten Kolorektalkarzinom eine Vielzahl von Einsatzmöglichkeiten für den monoklonalen Antikörper (nach C.H. Köhne, Dresden).

ligen Bronchialkarzinom. In beiden Indikationen sind die Ergebnisse laut Professor Dr. Hansjochen Wilke von der Internistischen Onkologie/Hämatologie des Klinikums Essen-Mitte viel versprechend, bedürfen aber noch der Überprüfung in größeren Untersuchungen.

\section{Zulassungsstatus}

Cetuximab ist seit Ende 2003 in der Schweiz unter dem Markennamen Erbitux $^{\mathrm{TM}}$ zur Behandlung des Irinotecan-refraktären Kolorektalkarzinoms zugelassen. Die amerikanische Zulassung erfolgte im Februar 2004. Bei der europäischen Zulassungsbehörde ist bereits ein Antrag eingereicht. Mit einem positiven Bescheid wird noch für dieses Jahr gerechnet.

\section{Quelle}

Satellitensymposium «Cetuximab - klinischer Fortschritt durch Anti-EGFR-Therapie bei soliden Tumoren» am 27. Februar 2004 im Rahmen des 26. Deutschen Krebskongress in Berlin

\begin{tabular}{l} 
Impressum \\
\hline Neue Therapieoption für solide Tumoren \\
PharmaForum in Onkologie 27 | 2 | 04 \\
\hline @ 2004 by S. Karger Verlag für Medizin und Natur- \\
wissenschaften GmbH \\
Lörracher Straße 16a \\
D-79115 Freiburg \\
Tel. +49 761452070 \\
E-mail Information@Karger.de \\
\hline
\end{tabular}

Mit freundlicher Unterstützung durch Merck KGaA, Darmstadt.

Der Verlag und die Herausgeber der Zeitschrift übernehmen keine Verantwortung für diese Rubrik.

Die Wiedergabe von Gebrauchsnamen, Handelsnamen, Warenbezeichnungen usw. in dieser Zeitschrift berechtigt auch ohne besondere Kennzeichnung nicht zur Annahme, dass solche Namen im Sinne der Warenzeichen- und Markenschutz-Gesetzgebung als frei zu betrachten wären und daher von jedermann benutzt werden dürfen. Für Angaben von Dosierungsanweisungen und Applikationsformen kann vom Verlag keine Haftung übernommen werden. Derartige Angaben müssen vom jeweiligen Anwender im Einzelfall anhand anderer Literaturstellen auf ihre Richtigkeit überprüft werden. 